\title{
Prompt and accurate diagnosis of ventricular arrhythmias with a novel index based on phase space reconstruction of ECG
}

\author{
George Koulaouzidis a,b,*, Saptarshi Das ${ }^{c}$, Grazia Cappiello ${ }^{c}$, Evangelos B. Mazomenos ${ }^{c}$, Koushik Maharatna ${ }^{\text {c }}$, \\ Paolo E. Puddu ${ }^{\text {d, John M. Morgan }}{ }^{a}$
}

a University Hospital Southampton NHS Foundation Trust, UK

b Department of Cardiology, Castle Hill Hospital, Hull York Medical School (at University of Hull), Kingston Upon Hull, UK

c School of Electronics and Computer Science, University of Southampton, UK

d Department of Cardiovascular Sciences, Sapienza University of Rome, Italy

\section{A R T I C L E I N F O}

\section{Article history:}

Received 9 November 2014

Accepted 21 December 2014

Available online 23 December 2014

\section{Keywords:}

Timely diagnosis

Ventricular arrhythmias

Index

Phase space reconstruction of ECG

\begin{abstract}
A B S T R A C T
Aim: To develop a statistical index based on the phase space reconstruction (PSR) of the electrocardiogram (ECG) for the accurate and timely diagnosis of ventricular tachycardia (VT) and ventricular fibrillation (VF). Methods: Thirty-two ECGs with sinus rhythm (SR) and 32 ECGs with VT/VF were analyzed using the PSR technique. Firstly, the method of time delay embedding were employed with the insertion of delay " $\tau$ " in the original time-series $X(t)$, which produces the $Y(t)=X(t-\tau)$. Afterwards, a PSR diagram was reconstructed by plotting $Y(\mathrm{t})$ against $X(\mathrm{t})$. The method of box counting was applied to analyze the behavior of the PSR trajectories. Measures as mean $(\mu)$, standard deviation $(\sigma)$ and coefficient of variation $(C V=\sigma / \mu)$, kurtosis $(\beta)$ for the box counting of PSR diagrams were reported.

Results: During SR, $C V$ was always $<0.05$, while with the onset of arrhythmia $C V$ increased $>0.05$. A similar pattern was observed with $\beta$, where $<6$ was considered as the cut-off point between SR and VT/VF. Therefore, the upper threshold for SR was considered $C V_{\text {th }}=0.05$ and $\beta_{t h}<6$. For optimisation of the accuracy, a new index $(J)$ was proposed: $J=w \frac{C V}{C V_{t h}}+(1-w) \frac{\beta}{\beta_{t h}}$.

During SR the upper limit of $J$ was the value of 1 . Furthermore $C V, \beta$ and $J$ crossed the cut-off point timely before the onset of arrhythmia (average time: $4 \mathrm{~min} 31 \mathrm{~s}$; SD: $2 \min 30 \mathrm{~s}$ ); allowing sufficient time for preventive therapy.

Conclusion: The J index improved ECG utility for arrhythmia monitoring and detection utility, allowing the prompt and accurate diagnosis of ventricular arrhythmias.
\end{abstract}

(c) 2014 Elsevier Ireland Ltd. All rights reserved.

\section{Introduction}

Sudden cardiac death (SCD), despite progress in epidemiological insight and effective therapeutic interventions, remains a major clinical and public health problem and one of the leading causes of mortality in the western world [1-3]. SCD can present without warning or a recognized triggering mechanism. Consequently, methodologies for prediction of SCD acquire a unique and critical importance for management of this public health issue. Despite active research in the prediction of SCD, the annual incidence of SCD ranges between 50 and 100 per 100,000 in the general population [4].

SCD is often the result of ventricular tachyarrhythmias, such as ventricular tachycardia (VT) or fibrillation (VF). Approximately one

\footnotetext{
* Corresponding author at: Department of Cardiology, Castle Hill Hospital, Hull York Medical School (at University of Hull), Kingston Upon Hull, UK.
}

third of these patients could be rescued with the timely use of a defibrillator [5,6]. Furthermore, it is of vital importance to differentiate VT from a stable but fast sinus rhythm (SR). Despite the established criteria, correct diagnosis of VT remains a challenge [7-9].

Phase-space reconstruction (PSR) is a technique, widely used in the field of nonlinear dynamics, for detecting small desynchronization phenomena in a time-series data and has found clinical application with several investigators [10-14]. Briefly, with the PSR technique a dynamic system's trajectory is reconstructed by plotting the original signal and its delayed version(s) along mutually orthogonal axes. This gives a closed contour for a periodic signal representing a limit cycle for the regular oscillations of the system under investigation. In healthy individuals, the consecutive ECG beats can be considered as almost periodic and therefore the PSR analysis produces an almost closed contour (Fig. 1). Therefore, the PSR technique has sensitivity for arrhythmia detection and identification of morphological changes in the ECG. 


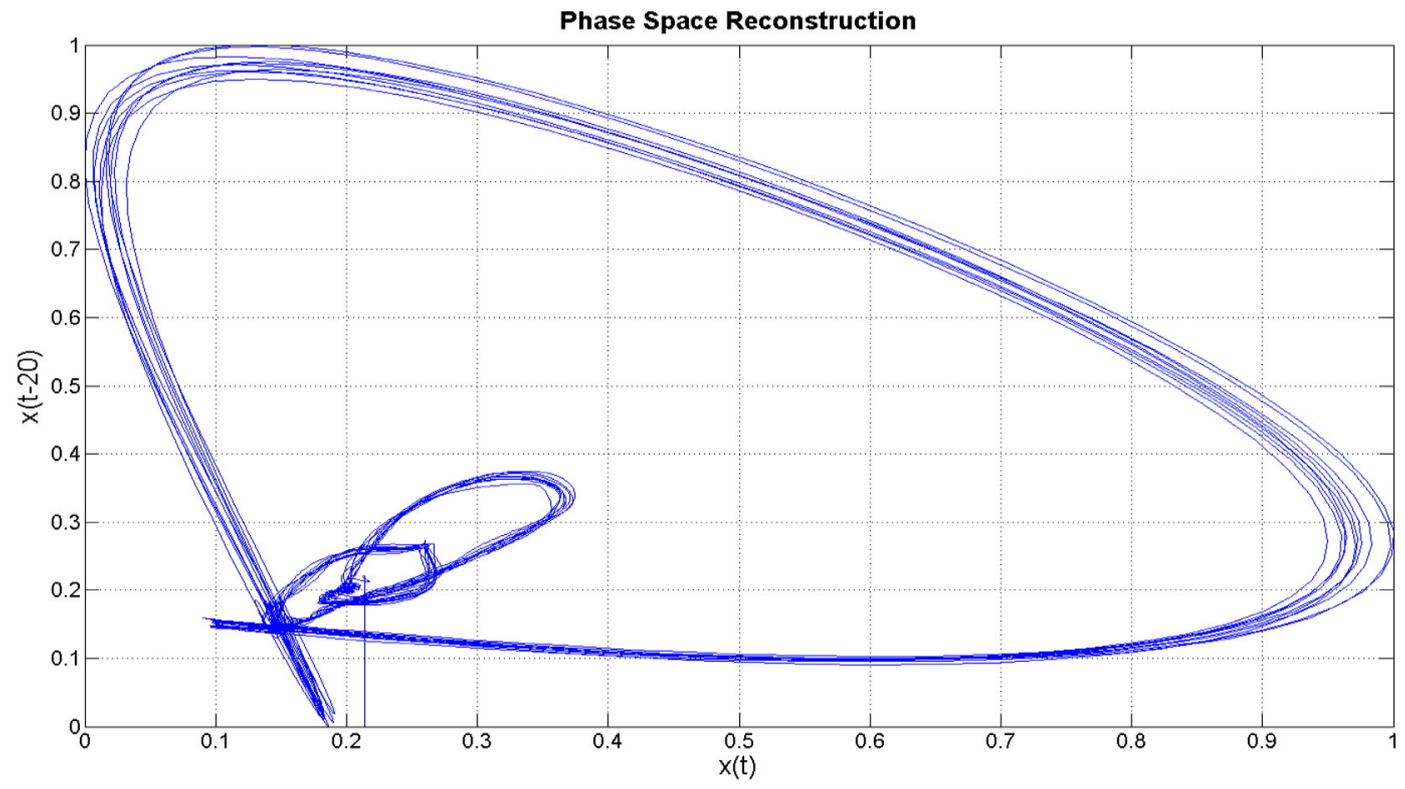

Fig. 1. Space phase reconstruction of 10 heart beats from a sinus rhythm ECG.

The purpose of this study was to develop a statistical index based on the PSR of the ECG for the accurate and timely diagnosis of ventricular arrhythmia.

\section{Methods}

Thirty-two ECGs from subjects with SR and 32 ECGs from subjects with VT/VF, available from the Physionet database, have been used in this study. Two expert cardiologists

$\mathrm{b}$

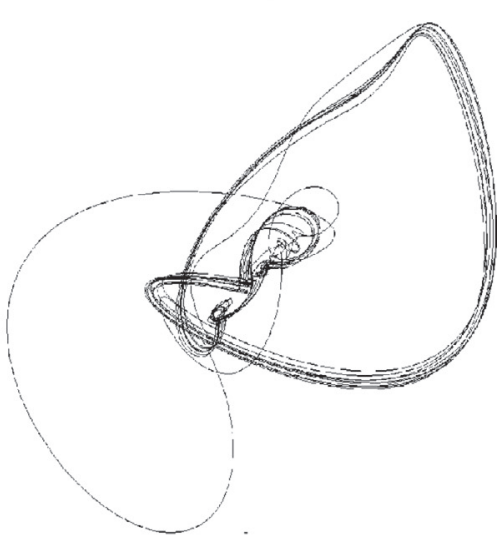

d B\&W Image

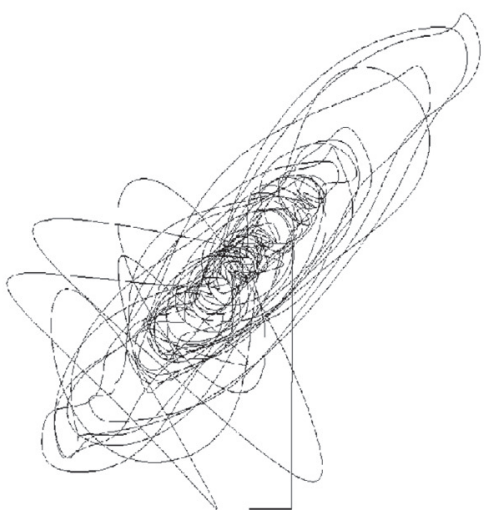

Fig. 2. Black and white image of a window of ten beats for: (a) SR on ECG, (b) SR with VPB on ECG, (c) VT on ECG, and (d) VF on ECG. 

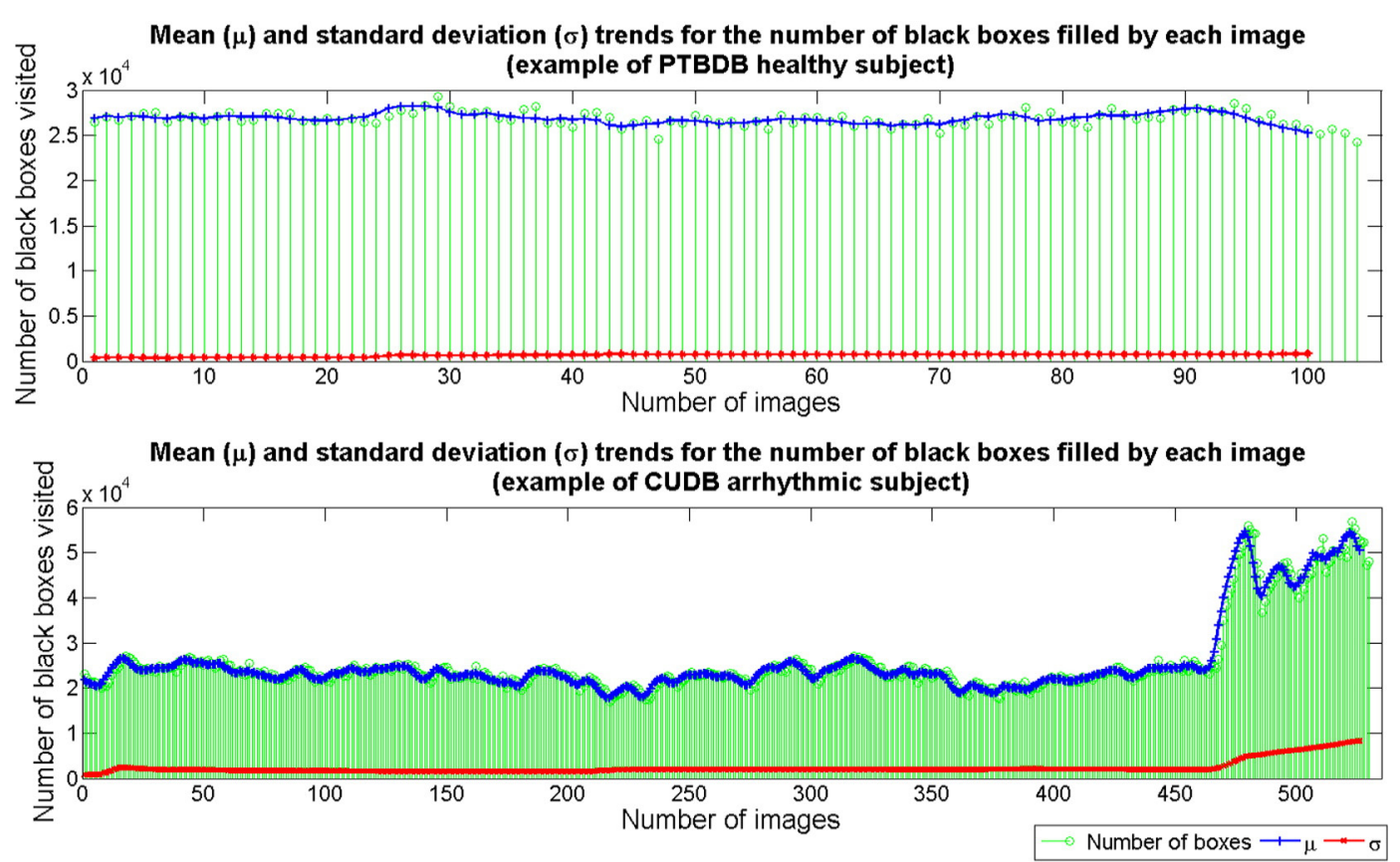

Fig. 3. Box counting mean and standard deviation trends for patient with healthy and arrhythmic patient respectively.

performed the selection of ECGs and the clinical annotation (identification of sinus rhythm, type of arrhythmia, abnormal ECG beats). For the analysis of ECG signals the boundaries of each heartbeat were detected reliably, with the use of automated time domain morphology and gradient (TDMG) algorithm.

For the PSR of the ECG, a window of 10 successive heartbeats was chosen. As PSR has improved accuracy if the signals are noise-free, all the ECG signals were filtered using a fourth-order Butterworth band-pass digital filter with the pass-band cut-off frequency of $1 \mathrm{~Hz}$ to $30 \mathrm{~Hz}$, to eliminate baseline wandering and high frequency measurement noise respectively. For the PSR, a 20 ms delay was applied in the filtered and normalized ECGs. A total of 10 trajectories were obtained from a window of 10 ECG beats in twodimensional (2-D) phase portraits, which was resized and then exported as a highresolution gray-scale image of pixel size $1024 \times 1024$ (Fig. 2).

Once the phase portrait is constructed, it is necessary to analyze the statistical behavior of the phase trajectories, as the number of trajectories and their spread. The wildly used technique of box counting was applied for that purpose. In this technique the entire phase portrait was represented as an image of $N \times N$ pixel, where $N$ is an integer. The pixels through which at least one trajectory has passed were considered as black boxes $\left(n_{b}\right)$ and the others were considered as white boxes $\left(n_{w}\right)$.

The statistical analysis of these phase portraits was carried out using Matlab where the black and white pixels were assigned as " 0 " and " 1 " values, respectively. Subsequently the number of black pixels was counted as they indicated the measure of spread of the trajectories and the underlying desynchronization phenomenon. The number of phase portrait windows was set to a value of 25 , while each phase portrait contained the characteristics of 10 ECG beats. In this way the accuracy of the histogram construction is ensured for the number of black boxes to calculate higher statistical moments (Fig. 3).

\subsection{Statistical analyses of phase portraits}

Statistical measures like mean $(\mu)$, standard deviation $(\sigma)$, skewness $(\gamma)$, kurtosis $(\beta)$ and coefficient of variation $(C V=\sigma / \mu)$ values of the number of black boxes visited by the

Trends for the proposed performance index $J=w \times C V / C V_{t h}+(1-w) \times \beta / \beta_{t h}$ with $w=0.5$ for PTBDB

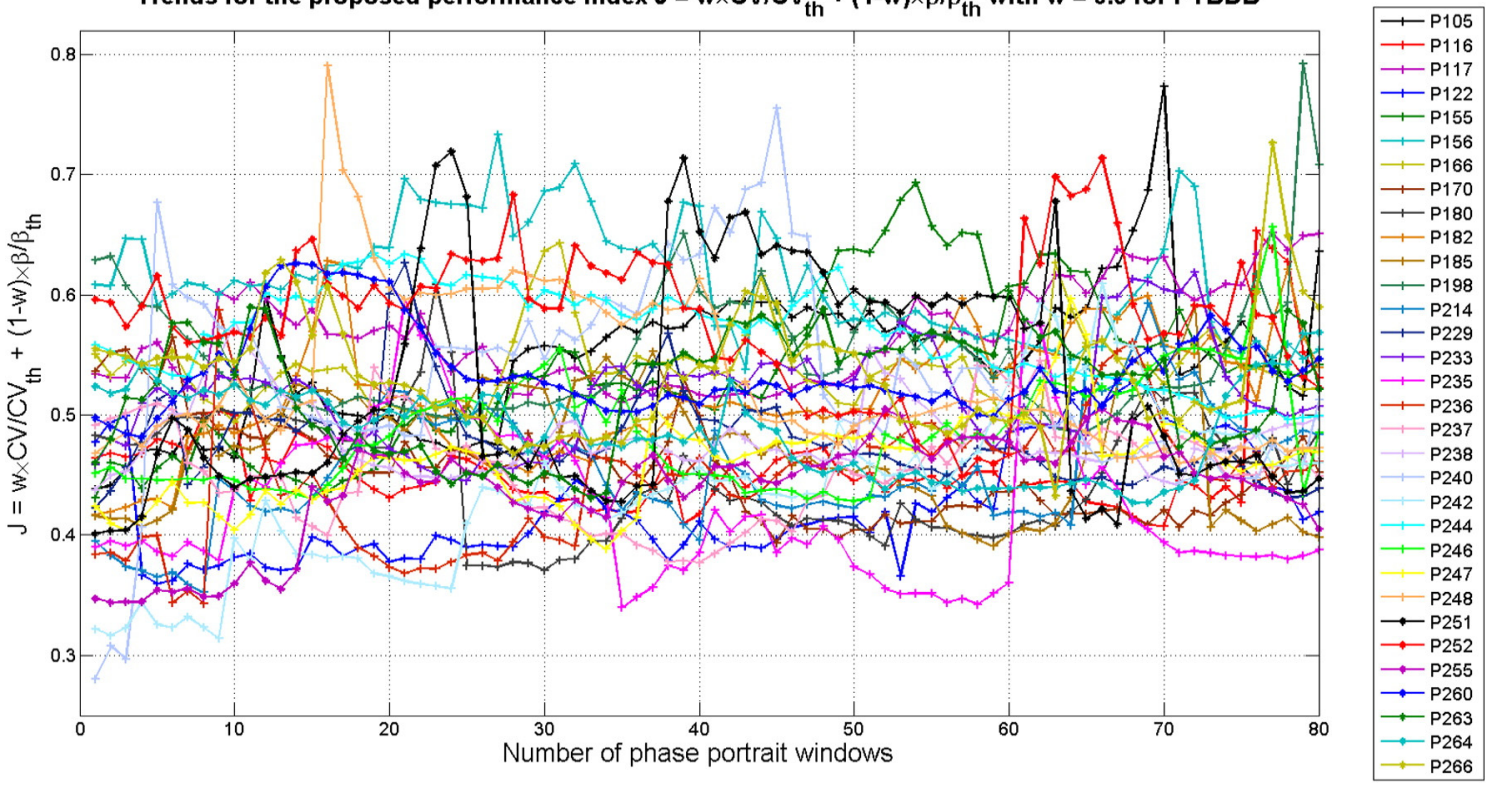

Fig. 4. Trends of $J$ index in subjects with SR. 
phase-space trajectories for each window of 25 ECG beats were estimated while their combinations were contributed in the development of the new index.

\section{Results}

In the 32 healthy subjects with SR, the analysis showed that $\mu$ and $\sigma$ trends are almost uniform throughout time. On the other hand, in the VT/VF subjects both $\mu$ and $\sigma$ showed sudden increase at the time of VT/VF onset. In order to identify the onset of arrhythmia accurately, $C V=\sigma / \mu$ trends were introduced. It has been observed that $C V$ is always bounded within an upper limit of $C V<0.05$, having values between $>0$ and $<0.05$. The arrhythmic subjects showed an increase in $C V$, which correlates with the onset of arrhythmia. During VT/VF the $C V$ remained stable above 0.05 . Therefore, the upper threshold was considered for the healthy subjects $C V_{\text {th }}=0.05$. A similar pattern was observed also with the kurtosis, in which despite the inter-person variability in each case, the kurtosis remained below an upper limit of $\beta_{t h}<6$ which was considered as the cut-off point between subjects with SR and VT/VF. In order to optimize the accuracy of our diagnosis a new index $(J)$ was proposed as a regularized and weighted combination of both the trends $\beta$ and $C V$ :

$J=w \frac{C V}{C V_{t h}}+(1-w) \frac{\beta}{\beta_{t h}}$.

The upper normal limit of index $J$ is the value of $J_{t h}=1$ while the crossing of upper bounds $C V_{t h}$ and $\beta_{t h}$ will be reflected in crossing of the threshold for the index of $J_{t h}=1$ (Fig. 4). In the above equation, the weight $w$ keeps the balance in the impact of $C V$ and $\beta$ trends in index $J$. So in healthy subjects, with equal weights on the two parts, $w$ would be 0.5 . In contrast in the VT/VF subjects, it is observed that the trends cross the critical threshold of $J=1$ at different time instants (Fig. 5). For $\mathrm{w}=0$ the full emphasis is on the kurtosis and with gradual increase of $w$, the impact of $C V$ increases slowly and consequently impact of kurtosis decreases. With $w=1$, the prediction index simply represents the $C V$ trend.
Fig. 6 shows the Receiver Operating Characteristics (ROC) curved for beta, J, and CV parameters. All parameters provide good results (b: AUC 0.92, 95\% Cl 0.82-0.97; J: AUC 0.96, 95\% Cl 0.89-0.99; CV: AUC $0.93 \mathrm{Cl}$ 0.84-0.98) with AUROC $>0.90$; however, the $J$ index performed the best, although no statistical difference is noted.

The new index was then tested to assess whether this approach enables the early identification of VA. In 29 subjects of the group with VA, $C V$ presented an acute and constant increase above the value of 0.05 (earlier than the actual arrhythmic event) and remained significantly increased until the VT/VF event. Thus the trajectory spread around the mean becomes more significant over time, indicating a desynchronization process in the physiological events leading to VT/VF. In these subjects during the time period, in which the increase in the $C V$ value is noted before the arrhythmic event, there are a minimum of 53 beats and a maximum of 827 beats, with an average of 356 beats with standard deviation of 192 beats (Fig. 7). In only 3 subjects, the CV crossed the cut-off value of 0.05 a short time (with $32,34,34$ beats) before the onset of VA. A further observation is that after the appearance of VPBs the value of the $J$ index raised significantly. In detail, VPBs occurred in 27 of subjects with VA and in all of them the $J$ index increased early and before the onset of VA. No VPBs were seen in the 3 subjects in whom the $J$ index was raised just before VA onset.

\section{Discussion}

The goal of this study was to quantify the PSR analysis of the ECG and introduce a novel statistical index for the diagnosis of VA. This hybrid index $(J)$ is a combination of coefficient of variation $(\mathrm{CV})$ and the kurtosis $(\beta)$ for the phase space diagrams of 10 beats. We identified that a threshold of $<0.05$ for $C V$, of $<6$ for $\beta$, and of $<1$ for $J$ to precisely differentiated VA from SR. Furthermore $C V, \beta$ and $J$ crossed the above threshold in individuals with VA promptly before the onset of that arrhythmia. The average time between the initial diagnostic increase of $C V, \beta$ and $J$ and the onset of VA was 4 min $31 \mathrm{~s}$ (SD: 2 min $30 \mathrm{~s}$ ) or 356 beats (SD: \pm 192 beats) while the minimum and the maximum time was $14 \mathrm{~s}$ and $8 \mathrm{~min}$
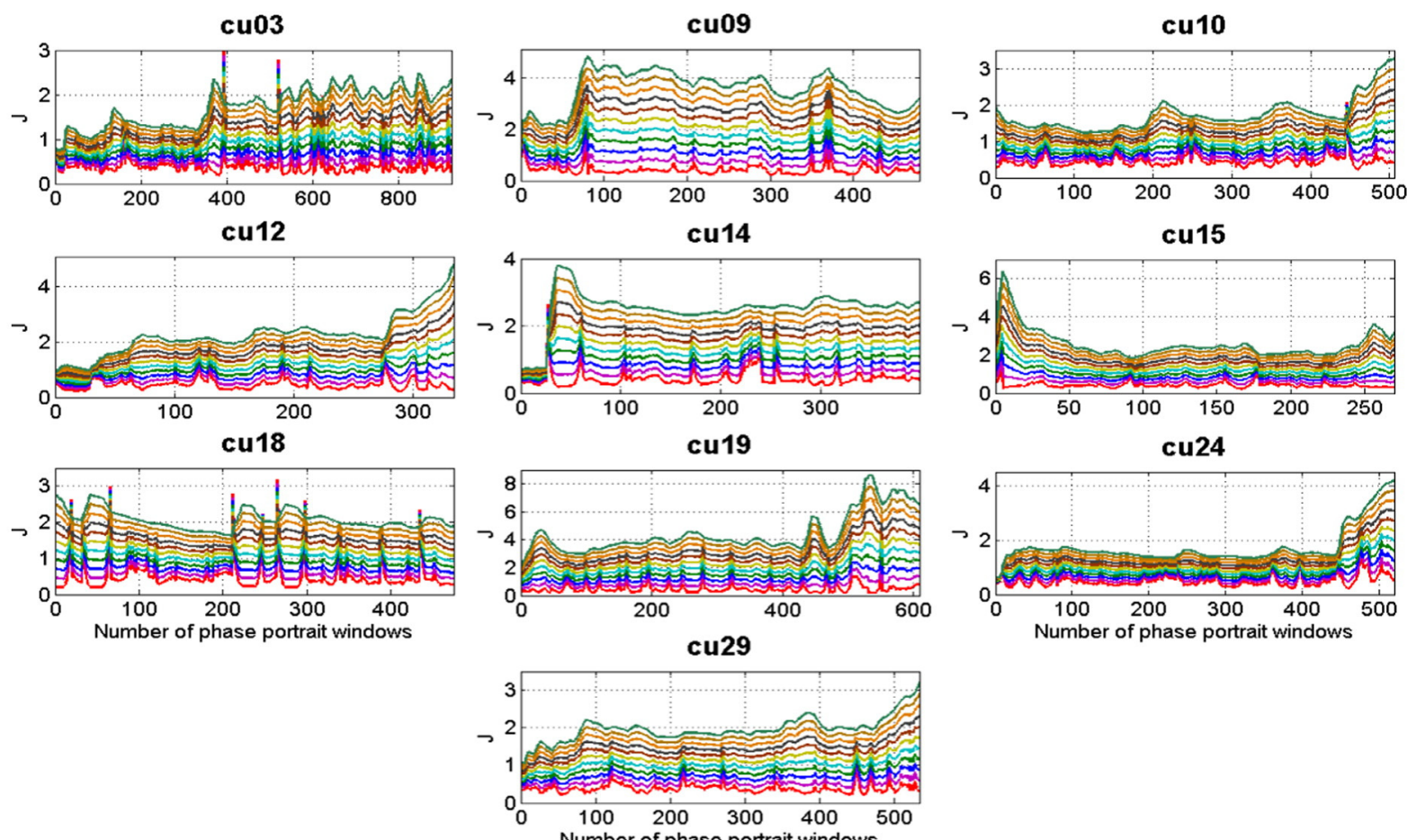

Number of phase portrait windows

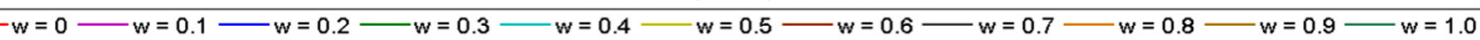

Fig. 5. Trend of $J$ index in subjects with VA. 


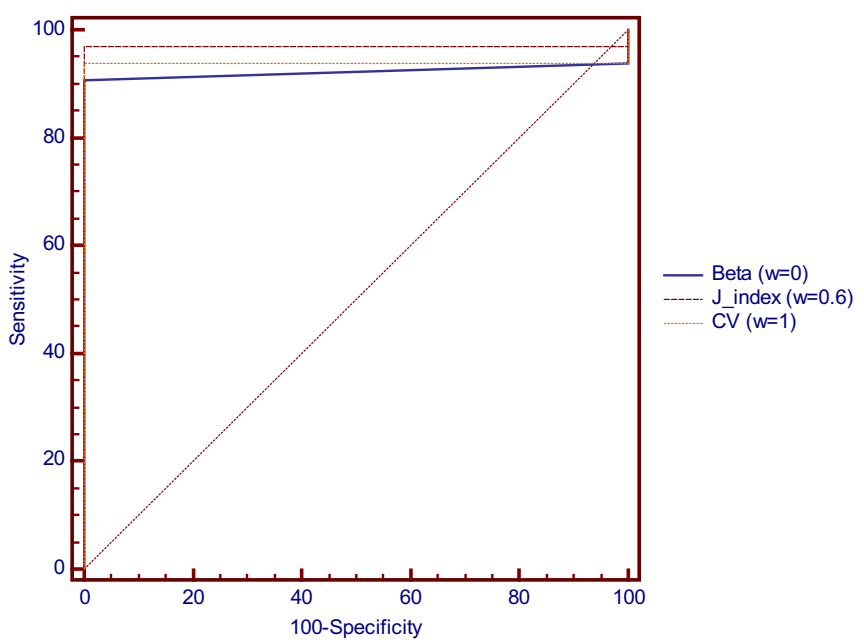

Fig. 6. Receiver operating characteristics (ROC) curved for Beta, $J$, and $C V$ parameters. $J$ parameter provides the best result, although beta and $C V$ also give an area under the ROC $($ AUROC $)>0.90$; no statistical difference is noted.

40 s respectively. Finally, VPBs were noted to play a crucial role in the triggering of VA.

The phase-space analysis technique has previously been used successfully as a method for the detection of coronary occlusion, the identification of ECG arrhythmias, analyses of QRS-complex time series, for distinguishing extrasystoles from normal heartbeats and for the understanding of heart rhythm dynamics [15-26]. However, in the above studies, the PSR method relies on visual examination of trajectories in PSR [27]. Visual assessment is subject to inter- and intra-observer variability that should be quantified if this method is to have practical application. To our knowledge, this is the first study to quantify the PSR analysis and propose a novel statistical index, which is easy to interpret.

VAs represent a common problem for physicians in every day clinical practice. They may present with a variety of clinical symptoms such as palpitations, chest pounding or long-lasting tachycardia accompanied with dyspnea, chest discomfort, hypotension and syncope. The most concern is directed towards the risk of SCD due to the unpredictable occurrence of sustained ventricular tachyarrhythmia, manifest on the ECG as VT or VF. In contrast to VF, monomorphic VT is a relatively “organized" tachyarrhythmia with discrete QRS complexes of constant morphology and must be differentiated from fast SR. Because of the hemodynamic consequences that accompany the onset of lethal VA, a preventive approach for treating VA is desirable. The ability to quickly identify and/or predict the impending onset of VA has major clinical value.

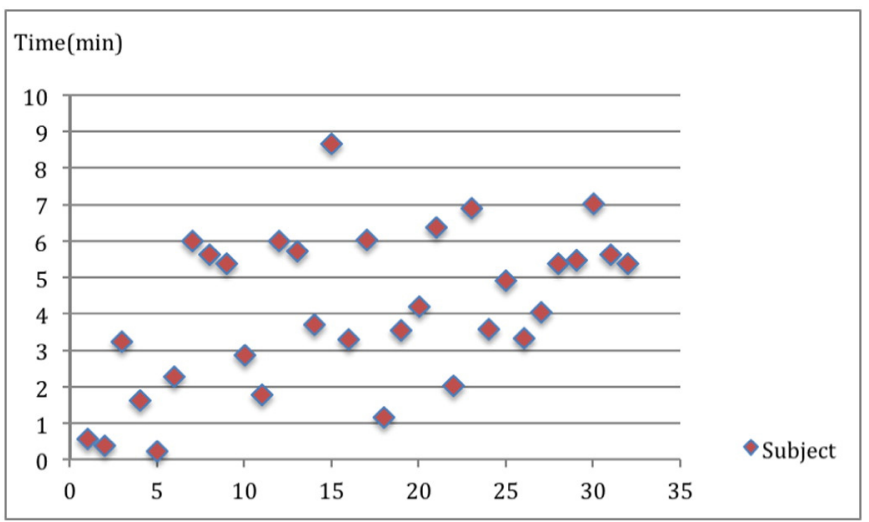

Fig. 7. The time difference (in $\mathrm{min}$ ) between the diagnosis of ventricular arrhythmia with the $J$ index and ECG in the 32 subjects.
The proposed $J$ index can diagnose these dangerous arrhythmias accurately and in a timely fashion so that it becomes a valuable clinical tool, and may have particular value for healthcare professionals with limited expertise in VA management. Furthermore, it may have value in the telemonitoring of patients with heart disease, as it will allow rapid analysis of a large volume of recordings. In addition, with prompt diagnosis of VA, the $J$ index can serve as an alert for clinicians for an imminent arrhythmia event. In the accident and emergency department or in the critical care unit, this will offer time to prepare therapeutic maneuvers which can be life saving. Finally, the $J$ index offers a mechanism for furthering investigation not triggering mechanisms for VAs [28-30].

\section{Limitations}

This is a pilot study based on ECG recordings already published electronically. A large-scale prospective study is needed to confirm the power of the proposed arrhythmia index in the clinical management of patients.

\section{Conclusion}

In this study, we developed a novel statistical index, " $J$ ", based on PSR of the ECG for the accurate and prompt diagnosis of VA. The index $J$ has potential to be a clinical tool for healthcare professionals with limited expertise VA management and also for critical care units where accurate and timely VA diagnosis is a necessity.

\section{Conflict of interest}

There is no conflict of interest.

\section{References}

[1] N.A. Mark Estes, III, Predicting and preventing sudden cardiac death, Circulation 124 (2011) 651-656.

[2] D.P. Zipes, H.J.J. Wellens, Sudden cardiac death, Circulation 98 (1998) 2334-2351.

[3] J.J1. Goldberger, A. Basu, R. Boineau, A.E. Buxton, M.E. Cain, J.M. Canty Jr., P.S. Chen, S.S. Chugh, O. Costantini, D.V. Exner, A.H. Kadish, B. Lee, D. Lloyd-Jones, A.J. Moss, R.J. Myerburg, J.E. Olgin, R. Passman, W.G. Stevenson, G.F. Tomaselli, W. Zareba, D.P. Zipes, L. Zoloth, Risk stratification for sudden cardiac death: a plan for the future, Circulation 129 (4) (2014) 516-526.

[4] R. Deo, C.M. Albert, Epidemiology and genetics of sudden cardiac death, Circulation 125 (2012) 620-637.

[5] R.M. John, U.B. Tedrow, B.A. Koplan, C.M. Albert, L.M. Epstein, M.O. Sweeney, A.L Miller, G.F. Michaud, W.G. Stevenson, Ventricular arrhythmias and sudden cardiac death, Lancet 380 (9852) (2012) 1520-1529.

[6] B.A. Koplan, W.G. Stevenson, Ventricular tachycardia and sudden cardiac death, Mayo Clin. Proc. 84 (3) (2009) 289-297.

[7] H.J.J. Wellens, Ventricular tachycardia: diagnosis of broad QRS complex tachycardia, Heart 86 (2001) 579-585.

[8] M.J. Griffith, P. Mounsey, A.J. Camm, C.J. Garratt, Ventricular tachycardia as default diagnosis in broad complex tachycardia, Lancet 343 (8894) (1994) 386-388.

[9] H. Hollowell, A. Mattu, A.D. Perron, C. Holstege, W.J. Brady, Wide-complex tachycardia: beyond the traditional differential diagnosis of ventricular tachycardia vs supraventricular tachycardia with aberrant conduction, Am. J. Emerg. Med. 23 (7) (2005) 876-889.

[10] M.I. Owis, A.H. Abou-Zied, A.M. Youssef, Y.M. Kadah, Study of features based on nonlinear dynamical modelling in ECG arrhythmia detection and classification, IEEE Trans. Biomed. Eng. 49 (2) (2002) 733-736.

[11] X.S. Zhang, Y.S. Zhu, X.J. Zhang, New approach to studies on ECG dynamics: extraction and analyses of QRS complex irregularity time series, Med. Biol. Eng. Comput. 35 (5) (1997) 467-473.

[12] M. Roopaei, R. Boostani, R.R. Sarvestani, M.A. Taghavi, Z. Azimifar, Chaotic based reconstructed phase space features for detecting ventricular fibrillation, Biomed. Signal Proc. Control 5 (4) (2010) 318-327.

[13] A. Amann, R. Tratnig, K. Unterkofler, Detecting ventricular fibrillation by time-delay methods, IEEE Trans. Biomed. Eng. 54 (1) (2007) 174-177.

[14] O. Fojt, J. Holcik, Applying nonlinear dynamics to ECG signal processing, IEEE Eng. Med. Biol. Mag. 17 (2) (1998) 96-101.

[15] J. Fell, K. Mann, J. Roschke, M.S. Gopinathan, Nonlinear analysis of continuous ECC during sleep I. Reconstruction, Biol. Cybern. 82 (6) (2000) 477-483.

[16] F.M. Roberts, R.J. Povinelli, K.M. Ropella, Identification of ECG arrhythmias using phase space reconstruction, 5th European Conference on Principles and Practice of Knowledge Discovery in Databases, 2001, pp. 411-423.

[17] M. Small, D.J. Yu, N. Grubb, J. Simonotto, K.A.A. Fox, R.G. Harrison, Automatic identification and recording of cardiac arrhythmia, Comput. Cardiol. 27 (2000) 355-358. 
[18] J. Fell, K. Mann, J. Roschke, M.S. Gopinathan, Nonlinear analysis of continuous ECG during sleep II. Reconstruction, Biol. Cybern. 82 (6) (2000) 485-491.

[19] H.L. Chan, S.C. Fang, P.K. Chao, C.L. Wang, J.D. Wei, Phase-space reconstruction of electrocardiogram for heartbeat classification, IFMBE Proc. 25 (4) (2010) 1234-1237.

[20] G. Dori, Y. Denekamp, S. Fishman, A. Rosenthal, B.S. Lewis, H. Bitterman, Evaluation of the phase-plane ECG as a technique for detecting acute coronary occlusion, Int J of Card 84 (2002) 167-170.

[21] T. Rocha, S. Paredes, P. de Carvalho, J. Herniques, M. Antunes, Phase space reconstruction approach for ventricular arrhythmias characterization, Conf Procc IEEE Eng Med Biol Soc 2008 (2008) 5470-5473.

[22] H. Yang, S.T.S. Bukkapatnam, T. Le, R. Komanduri, Identification of myocardial infarction using spatio-temporal heart dynamics, Med. Eng. Phys. 34 (2012) 485-497.

[23] G. Koulaouzidis, S. Das, G. Cappiello, E.B. Mazomenos, K. Maharatna, J. Morgan, A novel approach for the diagnosis of ventricular tachycardia based on phase space reconstruction of ECG, Int. J. Cardiol. 172 (1) (2014) e31-e33.

[24] E.C. Karvounis, M.G. Tsipouras, D.I. Fotiadis, Detection of fetal heart rate through 3-d phase space analysis from multivariate abdominal recordings, IEEE Trans. Biomed. Eng. 56 (5) (2009) 1394-1406.
[25] I. Nejadgholi, M.H. Moradi, F. Abdolani, Using phase space reconstruction for patient independent heartbeat classification in comparison with some benchmark methods, Comput. Biol. Med. 41 (6) (2011) 411-419.

[26] M.W. Zimmerman, R.J. Povinelli, M.T. Johnson, K.M. Ropella, A reconstructed phase space approach for distinguishing ischemic from non-ischemic ST changes using holter ECG data, Comput. Cardiol. 30 (2003) 243-246.

[27] R.R. Sarvestani, R. Boostani, M. Roopaei, VT and VF classification using trajectoriy analysis, Nonlinear Anlaysis: Theory, Methods \& Applications 71 (12) (2009) 55-61.

[28] H.L. Chan, C.L. Wang, S.C. Fanq, P.K. Chao, J.D. Wei, Recognition of ventricular extrasystoles over the reconstructed phase space of electrocardiogram, Ann. Biomed. Eng. 38 (3) (2010) 813-823.

[29] F. Santoro, L.D. Blase, P. Hrantitzky, J.E. Sanchez, P. Santageli, A.P. Perini, J.D. Burkhardt, A. Natale, Ventricular fibrillation triggered by PVCs from papillary muscles: clinical features and ablation, J. Cardiovasc. Electrophysiol. 25 (11) (2014) 1158-1165.

[30] J.A. Gomes, R.I. Hariman, P.S. Kanq, N. El-Sherif, I. Chowdhry, J. Lyons, Programmed electrical stimulation in patients with high-grade ventricular ectopy: electrophysiologic findings and prognosis for survival, Circulation 70 (1) (1984) 43-51. 